\title{
Proximity Effect and Multiple Andreev Reflections in Diffusive Superconductor-Normal-Metal-Superconductor Junctions
}

\author{
J.C. Cuevas ${ }^{1,2}$, J. Hammer ${ }^{1}$, J. Kopu ${ }^{1,3}$, J.K. Viljas ${ }^{1}$, and M. Eschrig ${ }^{1}$ \\ ${ }^{1}$ Institut für Theoretische Festkörperphysik, Universität Karlsruhe, 76128 Karlsruhe, Germany \\ ${ }^{2}$ Departamento de Física Teórica de la Materia Condensada, \\ Universidad Autónoma de Madrid, 28049-Madrid, Spain and \\ ${ }^{3}$ Low Temperature Laboratory, Helsinki University of Technology, P.O.Box 2200, FIN-02015 HUT, Finland
}

(Dated: February 2, 2008)

\begin{abstract}
We present a theory of the current-voltage characteristics in diffusive superconductor-normalmetal-superconductor junctions. By solving the time-dependent Usadel equations we are able to describe the phase-coherent transport for arbitrary length of the normal wire. We show how the interplay between proximity effect and multiple Andreev reflections gives rise to a rich subgap structure in the conductance and how it is revealed in the non-equilibrium distribution function.
\end{abstract}

PACS numbers: 74.45.+c,74.50.+r,73.23.-b

Proximity effect is the modification of the properties of a normal metal $(\mathrm{N})$ in contact with a superconductor (S) and it has been extensively studied in diffusive hybrid nanostructures 1]. Both equilibrium [2] and transport properties [3, 4] of diffusive $\mathrm{SN}$ systems are now well understood in the framework of the Usadel equations 5]. The transport through an SN interface is mediated by Andreev reflection, where an electron coming from $\mathrm{N}$ with energy $\epsilon$ below the superconducting gap $\Delta$ is converted into a reflected hole, thus transferring a Cooper pair to the $\mathrm{S}$ electrode. The time-reversed states involved in this process are coherent over a distance $L_{C}=\min \left(\sqrt{\hbar D / \epsilon}, L_{\phi}\right)$, where $D$ is the diffusion constant of $\mathrm{N}$ and $L_{\phi}$ is the phase coherence length.

In an SNS junction the transport at finite bias is dominated by multiple Andreev reflections (MARs). Here, successive Andreev reflections at both S electrodes lead to a progressive rise of the quasiparticle energy. This process continues until the quasiparticle energy exceeds the gap energy. A microscopic theory of MARs has emerged recently and has been shown to describe quantitatively the current-voltage (I-V) characteristics [ $[$ ], the noise [7] and the supercurrent [8] in atomic point contacts.

The interplay between proximity effect and MARs in diffusive SNS systems gives rise to a rich variety of physical phenomena. For instance, the conductance exhibits a very peculiar subgap structure 9, 10, 11]. This interplay is also revealed in the noise [1, 12] or in the Shapiro steps [13]. The understanding of these experiments is a basic open problem in mesoscopic superconductivity.

The theory of dissipative transport in diffusive SNS junctions has mainly been developed in two limits. The first one is the incoherent regime, when the normal metal length $L>L_{C}$. In this case there is no proximity effect and the transport can be described in terms of a semiclassical kinetic equation for the distribution function [14]. This function was actually measured in Ref. 15, and it was shown to exhibit a step-like structure, which is a manifestation of MARs. On the other hand, in short SNS junctions, when $L \ll \xi=\sqrt{\hbar D / \Delta}$, where $\xi$ is the superconducting coherence length, i.e. when the Thouless energy $\epsilon_{T}=\hbar D / L^{2}$ exceeds $\Delta$, the MARs are fully coherent. In this regime the transport can be described by averaging the single-channel point-contact results with the bimodal transmission distribution for diffusive systems [16]. For the intermediate regime $\xi<L<L_{\phi}$, when the interplay between proximity effect and MARs takes place [17], there is no satisfactory theory so far.

In this Letter we study the phase-coherent transport in diffusive voltage-biased SNS systems. We have solved the time-dependent Usadel equations, which allows us to calculate the I-V characteristics for the whole range of lengths from the short junction limit $(L \ll \xi)$ to the incoherent regime $(L \gg \xi)$. We show that the interplay between proximity effect and MARs gives rise to a rich structure in the conductance in good agreement with existing experiments. We also predict how this interplay is manifested in the quasiparticle distribution function.

We consider an SNS junction, where $\mathrm{N}$ is a diffusive normal metal of length $L<L_{\phi}$ coupled to two identical superconducting reservoirs. We assume the SN interfaces to be fully transparent and neglect the suppression of the pair potential in the $\mathrm{S}$ leads near the interfaces. Our goal is the calculation of the current when a constant voltage $V$ is applied. For this purpose we use the quasiclassical theory of superconductivity for diffusive systems [5]. This theory is formulated in terms of momentum averaged Green functions $\check{\mathbf{G}}\left(\mathbf{R}, t, t^{\prime}\right)$ which depend on position $\mathbf{R}$ and two time arguments. These propagators are $2 \times 2$ matrices in Keldysh space ( () , where each entry is a $2 \times 2$ matrix in electron-hole space $\left(^{\wedge}\right)$ :

$$
\check{\mathbf{G}}=\left(\begin{array}{cc}
\hat{G}^{R} & \hat{G}^{K} \\
0 & \hat{G}^{A}
\end{array}\right) ; \quad \hat{G}^{R}=\left(\begin{array}{cc}
\mathcal{G}^{R} & \mathcal{F}^{R} \\
\tilde{\mathcal{F}}^{R} & \tilde{\mathcal{G}}^{R}
\end{array}\right) .
$$

The Green functions for the left (l) and right (r) leads can be written as $\check{\mathbf{G}}_{l, r}\left(t, t^{\prime}\right)=e^{-i \mu_{l, r} t \hat{\tau}_{3} / \hbar} \check{\mathbf{G}}_{0}\left(t-t^{\prime}\right) e^{i \mu_{l, r} t^{\prime} \hat{\tau}_{3} / \hbar}$, where for the chemical potentials we use $\mu_{l}=0, \mu_{r}=e V$. Here, $\check{\mathbf{G}}_{0}(t)$ is the equilibrium bulk Green function of 
a BCS superconductor. We transform to energy representation, in which the propagator $\check{\mathbf{G}}\left(\mathbf{R}, \epsilon, \epsilon^{\prime}\right)$ depends on two energy arguments. It satisfies the non-stationary Usadel equation, which in the $\mathrm{N}$ region reads

$$
\frac{\hbar D}{\pi} \nabla(\check{\mathbf{G}} \circ \nabla \check{\mathbf{G}})+\epsilon \hat{\tau}_{3} \check{\mathbf{G}}-\check{\mathbf{G}} \hat{\tau}_{3} \epsilon^{\prime}=0,
$$

where $\hat{\tau}_{3}$ is the Pauli matrix in electron-hole space. The convolution product $\circ$ is defined as $(\check{\mathbf{A}} \circ \check{\mathbf{B}})\left(\epsilon, \epsilon^{\prime}\right)=$ $\int d \epsilon_{1} \check{\mathbf{A}}\left(\epsilon, \epsilon_{1}\right) \check{\mathbf{B}}\left(\epsilon_{1}, \epsilon^{\prime}\right)$. Equation (2) is supplemented by the normalization condition $\check{\mathbf{G}} \circ \breve{\mathbf{G}}=-\pi^{2} \delta\left(\epsilon-\epsilon^{\prime}\right) \check{\mathbf{1}}$.

In order to solve the Usadel equation it is convenient to use the time-dependent Riccati parametrization [18]. In this method the Green functions are parametrized in terms of scalar retarded (R) and advanced (A) coherence functions $\gamma^{R, A}\left(\mathbf{R}, \epsilon, \epsilon^{\prime}\right)$ and $\tilde{\gamma}^{R, A}\left(\mathbf{R}, \epsilon, \epsilon^{\prime}\right)$, and two distribution functions $x\left(\mathbf{R}, \epsilon, \epsilon^{\prime}\right)$ and $\tilde{x}\left(\mathbf{R}, \epsilon, \epsilon^{\prime}\right)$ as follows

$$
\begin{aligned}
\hat{G}^{R} & =-i \pi \hat{N}^{R} \circ \hat{M}^{R}, \quad \hat{G}^{A}=i \pi \hat{M}^{A} \circ \hat{N}^{A} \\
\hat{G}^{K} & =-2 \pi i \hat{N}^{R} \circ \hat{M}^{K} \circ \hat{N}^{A}
\end{aligned}
$$

with the abbreviations

$$
\begin{aligned}
\hat{M}^{R, A} & =\left(\begin{array}{cc}
1-\gamma^{R, A} \circ \tilde{\gamma}^{R, A} & 2 \gamma^{R, A} \\
2 \tilde{\gamma}^{R, A} & \tilde{\gamma}^{R, A} \circ \gamma^{R, A}-1
\end{array}\right) \\
\hat{M}^{K} & =\left(\begin{array}{cc}
x+\gamma^{R} \circ \tilde{x} \circ \tilde{\gamma}^{A} & x \circ \gamma^{A}-\gamma^{R} \circ \tilde{x} \\
\tilde{\gamma}^{R} \circ x-\tilde{x} \circ \tilde{\gamma}^{A} & \tilde{x}+\tilde{\gamma}^{R} \circ x \circ \gamma^{A}
\end{array}\right) \\
\hat{N}^{R, A} & =\left(\begin{array}{cc}
1+\gamma^{R, A} \circ \tilde{\gamma}^{R, A} & 0 \\
0 & 1+\tilde{\gamma}^{R, A} \circ \gamma^{R, A}
\end{array}\right)^{-1},
\end{aligned}
$$

where the inverse is defined via the o-operation. Using fundamental symmetries, all the Green functions can be obtained from $\gamma^{R}$ and $x$. The transport equations for these functions in the $\mathrm{N}$ wire are 19]

$$
\begin{aligned}
\partial_{z}^{2} \gamma^{R}+ & \left(\partial_{z} \gamma^{R}\right) \circ \frac{\tilde{\mathcal{F}}^{R}}{i \pi} \circ\left(\partial_{z} \gamma^{R}\right)=\frac{\mathcal{E} \circ \gamma^{R}+\gamma^{R} \circ \mathcal{E}}{i \epsilon_{T}} \\
\partial_{z}^{2} x-\left(\partial_{z} \gamma^{R}\right) \circ \frac{\tilde{\mathcal{G}}^{K}}{i \pi} \circ\left(\partial_{z} \tilde{\gamma}^{A}\right) & +\left(\partial_{z} \gamma^{R}\right) \circ \frac{\tilde{\mathcal{F}}^{R}}{i \pi} \circ\left(\partial_{z} x\right) \\
-\left(\partial_{z} x\right) \circ \frac{\mathcal{F}^{A}}{i \pi} \circ\left(\partial_{z} \tilde{\gamma}^{A}\right) & =\frac{\mathcal{E} \circ x-x \circ \mathcal{E}}{i \epsilon_{T}}
\end{aligned}
$$

where $\mathcal{E}\left(\epsilon, \epsilon^{\prime}\right) \equiv \epsilon \cdot \delta\left(\epsilon-\epsilon^{\prime}\right)$. Here, $0<z<1$ is the dimensionless coordinate which describes the position along the $\mathrm{N}$ wire. The expressions for $\tilde{\mathcal{F}}^{R}, \mathcal{F}^{A}$, and $\tilde{\mathcal{G}}^{K}$ are obtained by comparing Eq. (11) with Eq. (3). A solution of these equations can be found using the Ansatz:

$$
\gamma^{R}\left(\epsilon, \epsilon^{\prime}\right)=\sum_{m} \gamma_{0, m}^{R}(\epsilon) \delta\left(\epsilon-\epsilon^{\prime}+\omega_{m}\right)
$$

where $\omega_{m} \equiv 2 m e V$. Other Fourier components are defined via $\gamma_{n, m}^{R}(\epsilon)=\gamma_{0, m-n}^{R}\left(\epsilon+\omega_{n}\right)$. Using the above Ansatz for any two functions $A$ and $B$, the Fourier components of $(A \circ B)\left(\epsilon, \epsilon^{\prime}\right)$ are given by $[A \circ B]_{n, m}(\epsilon)=$ $\sum_{l} A_{n, l}(\epsilon) B_{l, m}(\epsilon)$. The equations for the Fourier components $\gamma_{n, m}^{R}(\epsilon)$ and $x_{n, m}(\epsilon)$ are the same as Eqs. (415),

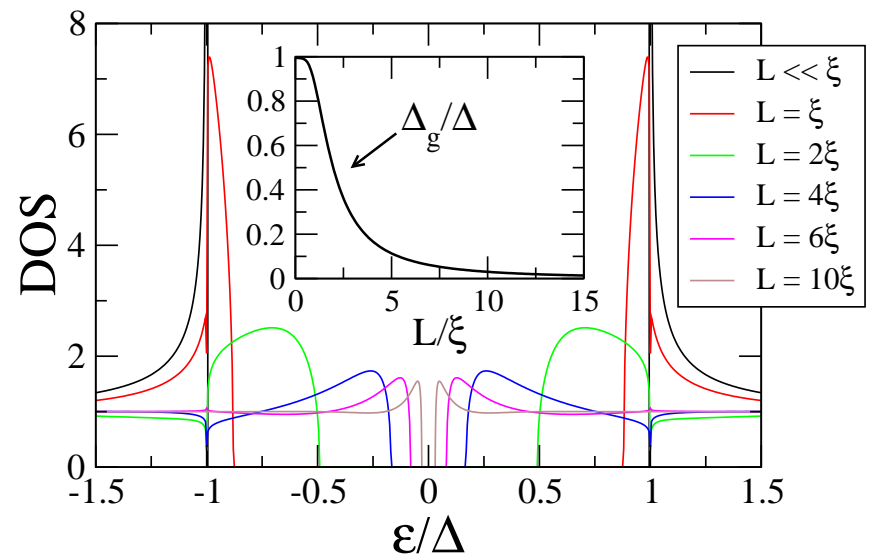

FIG. 1: (Color online) Normalized density of states in the middle of the wire as a function of energy for different wire lengths. Inset: minigap $\Delta_{g}$ as a function of the wire length.

where the o-product denotes now a matrix product in the Fourier indices and $\mathcal{E}_{n, m}(\epsilon)=\left(\epsilon+\omega_{n}\right) \cdot \delta_{n, m}$. The boundary conditions for Eqs. (45) can be easily deduced from the expressions of the bulk Green functions [20]. In general, these equations have to be solved numerically.

The above Ansatz leads to a current of the form $I(t)=$ $\sum_{m} I_{m} \exp \left(i m \omega_{J} t\right)$, where $\omega_{J}=2 \mathrm{eV} / \hbar$ is the Josephson frequency, and the current components can be written in terms of the Fourier components of the Green functions, $\check{\mathbf{G}}_{n, m}(\epsilon)$. We concentrate here on the analysis of the dc current $I_{0}$, which we shall denote as $I$. It reads

$$
I=\frac{G_{N}}{8 \pi^{2} e} \int_{0}^{2 e V} d \epsilon \sum_{m} \operatorname{Tr}\left\{\hat{\tau}_{3}\left[\check{\mathbf{G}} \circ \partial_{z} \check{\mathbf{G}}\right]_{m, m}^{K}(\epsilon)\right\},
$$

where $G_{N}$ is the normal state conductance. Next we express $I$ in terms of the distribution function. It is possible to relate the component $\hat{M}_{11}^{K}$ to the electron distribution function $f\left(\mathbf{R}, \epsilon, \epsilon^{\prime}\right)$ via the relation $2 f=$ $1-\sum_{n=0}^{\infty}\left(\gamma^{R} \circ \tilde{\gamma}^{R}\right)^{n} \circ \hat{M}_{11}^{K} \circ\left(\gamma^{A} \circ \tilde{\gamma}^{A}\right)^{n}$. Combining this with fundamental symmetries of the Green functions we can write the current as

$$
I=\frac{G_{N}}{e} \int_{0}^{2 e V} d \epsilon \sum_{m}\left\{\left[\mathcal{D} \circ \partial_{z} f\right]_{m, m}-\operatorname{Re}[\mathcal{S} \circ f]_{m, m}\right\}
$$

where $\mathcal{D}=1 / 2+\left(\mathcal{G}^{A} \circ \mathcal{G}^{R}-\mathcal{F}^{A} \circ \tilde{\mathcal{F}}^{R}\right) / 2 \pi^{2}$ and $\mathcal{S}=$ $\left(\mathcal{G}^{R} \circ \partial_{z} \mathcal{G}^{R}+\mathcal{F}^{R} \circ \partial_{z} \tilde{\mathcal{F}}^{R}\right) / \pi^{2}$. Here, $\mathcal{D}$ describes the renormalization of the diffusion constant and $\mathcal{S}$ describes the spectral supercurrent.

It is instructive to first have a look at the zero-bias density of states (DOS). In Fig. 1 we show the DOS in the middle of the wire for different values of $L$. The most prominent feature is the presence of a minigap $\Delta_{g}$ which scales with the wire length as shown in the inset of Fig. 1 In the limit of long wires we obtain $\Delta_{g} \approx 3.2 \epsilon_{T}$. It is worth remarking that while the DOS changes along the wire, the minigap is independent of the position. 


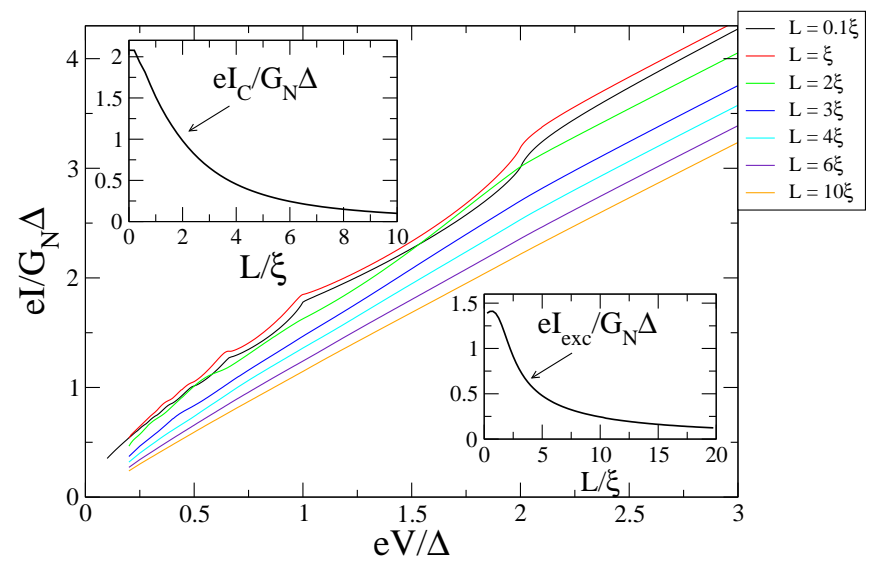

FIG. 2: (Color online) Zero-temperature dc current as a function of the voltage for different wire lengths $L$. Upper inset: zero temperature critical current as a function of $L$. Lower inset: excess current as a function of $L$.

Let us now turn to the analysis of the I-V characteristics. Figure 2 shows the zero-temperature I-V curves for different wire lengths. The main features are: (i) for $L \leq \xi$ there is a pronounced subharmonic gap structure (SGS) at voltages $2 \Delta / n e$ ( $n$ integer). In particular, the curve $L=0.1 \xi$ reproduces quantitatively the result of the short-junction limit [16]. (ii) For $L>\xi$ the SGS is progressively washed out as $L$ increases. (iii) At high bias $(\mathrm{eV}>>\Delta)$ there is an excess current, which is defined as $I_{e x c}=I-G_{N} V$. In the lower inset of Fig. 2 we show $I_{e x c}$ as a function of $L$. For $L \rightarrow 0$ we recover the result $e I_{\text {exc }} /\left(G_{N} \Delta\right)=\pi^{2} / 4-1$ of Ref. 16. In the opposite limit of long junctions $(L \gg \xi)$ Volkov et al. 21] found that $I_{e x c}$ decays according to $e I_{e x c} /\left(G_{N} \Delta\right)=0.82 \xi / L$. We find that $I_{e x c}$ can be fitted to $e I_{e x c} /\left(G_{N} \Delta\right)=2.47 \xi / L$ in the experimentally relevant range $3 \xi<L<20 \xi$, a factor of three larger than in the limit of Ref. 21.

As seen in Fig. 2] it is numerically difficult to reach the zero-bias limit, because the dimension of the matrices in Eqs. (46) scales with the voltage roughly as $(2 \Delta / \mathrm{eV})^{2}$. However, the analysis of the low bias regime is not the most relevant. The SNS junctions usually have a negligible capacitance and their I-V curves are hence nonhysteretic, exhibiting a transition from a supercurrent to a voltage state at the critical current. In the upper inset of Fig. 2 we show the value of the zero-temperature critical current, $I_{C}$. As can be seen, the transition to the supercurrent branch would take place at voltages which are accessible to our numerical solution.

The non-linearities in the I-Vs can more clearly be seen in the differential conductance, $G=d I / d V$, which is shown in Fig. 3 Notice that for $L \leq \xi$ the SGS consists of a set of pronounced maxima at roughly $e V_{n}=2 \Delta / n$ ( $n$ integer). For $L>\xi$ the shape and height of the maxima change drastically and new structure appears. For instance, the peak $n=2$ appears slightly below $\mathrm{eV}=\Delta$
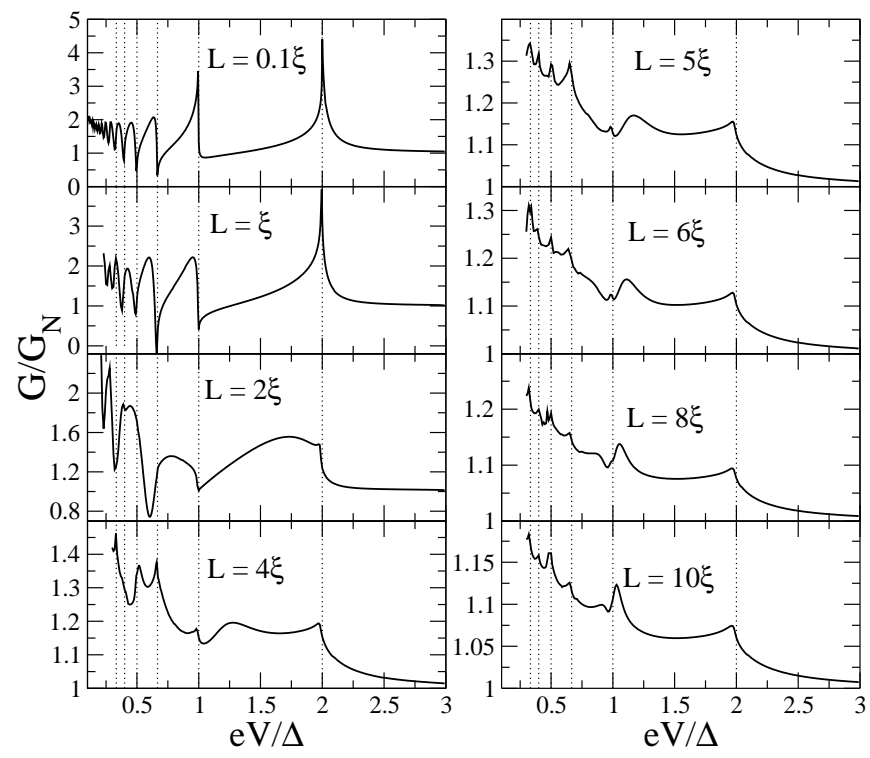

FIG. 3: Zero-temperature differential conductance as a function of the voltage for different wire lengths. The vertical lines indicate the position of $e V=2 \Delta / n$ with $n=1, \ldots, 6$.

and it is accompanied by a much more pronounced maximum above $\Delta$. This maximum shifts towards $\Delta$ as $L$ increases, until it merges with the peak at $\Delta$. The peak above $\Delta$ is a common feature of the experimental observations of the SGS 9, 10, 11]. Notice also that in the range $4 \xi<L<10 \xi$ the SGS is superimposed on a background that increases as the voltage decreases. The correction in the conductance (as compared to $G_{N}$ ) diminishes as $L$ increases and, for instance, it reaches 15\%-20\% at low bias for $L=10 \xi$. All these features are in qualitative agreement with the experimental observations (see e.g. Fig. 5 in Ref. 11). However, a quantitative comparison would require us to extend our analysis to arbitrary transparency of the SN interfaces.

The existence of the minigap $\Delta_{g}$ suggests that the SNS junction should exhibit a subgap structure similar to that of contacts with two different gaps 17]. Indeed, the conductance maximum above $\Delta$ appears roughly at $e V \sim \Delta+\Delta_{g}$. However, one must be careful with this analogy. First, other features expected for asymmetric junctions, like a peak at $\Delta-\Delta_{g}$, are absent. Moreover, our calculations show that as a function of temperature the conductance maxima shift following the bulk gap and, in particular, the peak above $\Delta$ survives even for temperatures higher than the minigap. The reason for the failure of the above argument is that at finite bias the minigap does not survive, and only a position-dependent pseudogap appears in the spectrum.

In Fig. 4 we present our results for the distribution function obtained for the intermediate regime, and compare with the results for the incoherent regime of Ref. 15 . We first summarize the main results of the incoherent 

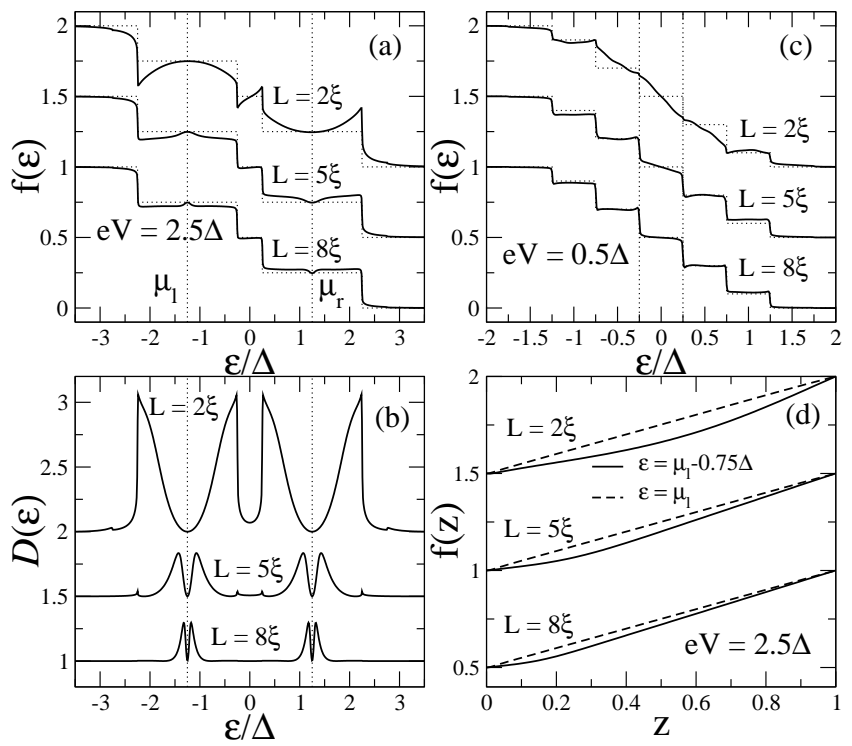

FIG. 4: (a) dc component of the distribution function in the middle of the wire $(z=1 / 2)$ for three different wire lengths $L$ and $e V=2.5 \Delta$. The position of the chemical potentials are indicated by vertical lines. The dotted lines are the results of the incoherent model (see text). (b) dc component of the renormalization of the diffusion constant for the cases shown in panel (a). (c) The same as panel (a) for $e V=0.5 \Delta$. (d) Spatial variation along the wire of the distribution functions in panel (a) for fixed energies. The curves in (a)-(d) have been shifted by multiples of 0.5 for convenience.

model discussed in Ref. 15. The basic assumptions are: (i) there is no proximity effect, and (ii) the probability of Andreev reflection is 1 within the gap and 0 outside. As a result, the dc component of $f$ varies linearly with position $z$. This model predicts a staircase pattern, and two examples are shown as dotted lines in Fig. 目(a,c), where the dc component of $f$ in the middle of the wire is plotted. Our full calculation shows pronounced deviations from this staircase pattern. An example can be seen in the energy region $-2.25 \Delta<\epsilon<-0.25 \Delta$ in Fig. 四(a), where $\mathrm{eV}=2.5 \Delta$. In this region, the incoherent model predicts $f=3 / 4$, while the full calculation gives smaller values in the whole window, except exactly at $\epsilon=\mu_{l}$.

We explain these deviations in terms of the proximity effect. As shown in Fig. 廿(d) for $e V=2.5 \Delta$, the proximity effect leads to a non-linear spatial variation of the distribution function $f$, except at the chemical potentials $\mu_{l, r}$. For $\epsilon=\mu_{l}$ the distribution function varies linearly from 0.5 to 1 , as in the incoherent model. However, for $\epsilon=\mu_{l}-0.75 \Delta$ the proximity effect near the left electrode leads to an effective shortening of the wire length. This explains the negative deviation for the electron distribution function in this energy range. For an energy near $\mu_{r}$ the deviation is positive, as the distribution function for this case (not shown here) varies from 0 to 0.5 and the proximity effect takes place near $z=1$.
A related aspect is the renormalization of the diffusion constant $\mathcal{D}$, the dc part of which is shown in Fig. 4(b). It reflects the enhancement of the transmission with respect to the normal state due to the proximity effect. Notice the correlation between the structure in $\mathcal{D}$ and the deviations of $f$ from the staircase pattern. In particular, at the chemical potentials of the superconductors $\mathcal{D}=1$, and $f$ adopts the values predicted by the incoherent model.

In summary, we have presented a theory of the I-V characteristics of diffusive SNS systems. We have studied how the interplay between coherent MARs and the proximity effect is reflected in the conductance and in the distribution function. Our main results are: (i) we reproduce an additional peak above $\Delta$ in the conductance, in agreement with experiments [9, 10, 11]; (ii) we predict the distribution function for the intermediate regime, which can be measured e.g. by the technique presented in Ref. 15. Our work paves the way for the study of other transport properties in diffusive SNS systems [12, 13].

We acknowledge fruitful discussions with D. Esteve, A. Levy Yeyati, A. Martín-Rodero, H. Pothier, and G. Schön. This work has been financed by the Helmholtz Gemeinschaft and by the DFG within the CFN.

[1] B. Pannetier and H. Courtois, J. Low Temp. Phys. 188, 599 (2000).

[2] S. Guéron et al., Phys. Rev. Lett. 77, 3025 (1996); N. Moussy et al., Europhys. Lett. 55, 861 (2001).

[3] H. Courtois et al., J. Low Temp. Phys. 116, 187 (1999).

[4] P. Dubos et al., Phys. Rev. B 63, 064502 (2001).

[5] K.D. Usadel, Phys. Rev. Lett. 25, 507 (1970).

[6] E. Scheer et al., Nature 394, 154 (1998).

[7] R. Cron et al., Phys. Rev. Lett. 86, 4104 (2001).

[8] M.F. Goffman et al., Phys. Rev. Lett. 85, 170 (2000).

[9] J. Kutchinsky et al., Phys. Rev. Lett. 78, 931 (1997); J. Kutchinsky et al., Phys. Rev. B 56, R2932 (1997).

[10] R. Taboryski et al., Superlattices Microstruct. 25, 829 (1999); J. Kutchinsky, Ph.D. thesis, Technical University of Denmark, 2001.

[11] T. Hoss et al., Phys. Rev. B 62, 4079 (2000).

[12] C. Hoffmann et al., Phys. Rev. B 70, 180503 (2004).

[13] P. Dubos et al., Phys. Rev. Lett. 87, 206801 (2001).

[14] E.V. Bezuglyi et al., Phys. Rev. B 62, 14439 (2000); E.V. Bezuglyi et al., Phys. Rev. B 63, 100501 (2001); K.E. Nagaev, Phys. Rev. Lett. 86, 3112 (2001); S. Pilgram and P. Samuelsson, Phys. Rev. Lett. 94, 086806 (2005).

[15] F. Pierre et al., Phys. Rev. Lett. 86, 1078 (2001).

[16] A. Bardas and D. Averin, Phys. Rev. B 56, 8518 (1997); A.V. Zaitsev and D.V. Averin, Phys. Rev. Lett. 80, 3602 (1998).

[17] P. Samuelsson et al., Phys. Rev. B 65, 180514 (2002).

[18] M. Eschrig, Phys. Rev. B 61, 9061 (2000).

[19] M. Eschrig et al., Adv. in Solid State Phys. 44, 533 (2004).

[20] $\left[x_{n, m}\right]_{l}(\epsilon)=x_{0}\left(\epsilon+\omega_{n}\right) \delta_{n, m},\left[\gamma_{n, m}^{R}\right]_{l}(\epsilon)=\gamma_{0}^{R}\left(\epsilon+\omega_{n}\right) \delta_{n, m}$, $\left[x_{n, m}\right]_{r}(\epsilon)=x_{0}\left(\epsilon+\omega_{n}+e V\right) \delta_{n, m},\left[\gamma_{n, m}^{R}\right]_{r}(\epsilon)=\gamma_{0}^{R}\left(\epsilon+\omega_{n}+\right.$ $e V) \delta_{n, m-1}$, where $\gamma_{0}^{R}(\epsilon)=-\Delta /\left\{\epsilon+i \sqrt{\Delta^{2}-\left(\epsilon+i 0^{+}\right)^{2}}\right\}$ 
and $x_{0}(\epsilon)=\tanh (\epsilon / 2 T) \cdot\left(1-\left|\gamma_{0}^{R}(\epsilon)\right|^{2}\right)$.

[21] A.F. Volkov et al., Physica C 210, 21 (1993). 